AperTO - Archivio Istituzionale Open Access dell'Università di Torino

UiO-66 type MOFs with mixed-linkers - 1,4-Benzenedicarboxylate and 1,4naphthalenedicarboxylate: Effect of the modulator and post-synthetic exchange

This is a pre print version of the following article:

Original Citation:

Availability:

This version is available http://hdl.handle.net/2318/1766094

since 2021-01-08T13:15:03Z

Published version:

DOI:10.1016/j.micromeso.2020.110324

Terms of use:

Open Access

Anyone can freely access the full text of works made available as "Open Access". Works made available under a Creative Commons license can be used according to the terms and conditions of said license. Use of all other works requires consent of the right holder (author or publisher) if not exempted from copyright protection by the applicable law. 


\title{
UiO-66 type MOFs with mixed-linkers - 1,4-Benzenedicarboxylate and 1,4- Naphthalenedicarboxylate: effect of the modulator and post-synthetic exchange
}

Vera V. Butova*1, Olga A. Burachevskaya ${ }^{1}$, Ilya V. Ozhogin ${ }^{2}$, Gennady S. Borodkin ${ }^{2}$, Andrey G. Starikov ${ }^{2}$, Silvia Bordiga ${ }^{3}$, Alessandro Damin ${ }^{3}$, Karl Petter Lillerud $^{4}$, Alexander V. Soldatov ${ }^{1}$

${ }^{1}$ The Smart Materials Research Institute, Southern Federal University, Sladkova 178/24, Rostov-on-Don, 344090, Russian Federation

${ }^{2}$ Institute of Physical and Organic Chemistry, Southern Federal University, 194/2 prosp. Stachki, Rostovon-Don, 344090, Russian Federation

${ }^{3}$ Department of Chemistry, NIS Interdepartmental Centre and INSTM Reference Centre, University of Turin, via Quarello 15A, I-10135 Turin, Italy

${ }^{4}$ Centre for Materials Science and Nanotechnology, Department of Chemistry, University of Oslo, Sem Saelandsvei 26, N-0315 Oslo, Norway

Vera V. Butova: vbutova@sfedu.ru

\begin{abstract}
We present a detailed study of the effect of benzoic acid as a modulator on the properties of UiO66 type MOFs with two linkers 1,4-benzenedicarboxylate (BDC) and 1,4naphthalenedicarboxylate (NDC). We used four compositions of MOFs with molar ratios BDC/NDC 100/0, 75/25, 50/50, and 0/100. For each composition effect of benzoic acid (BA) additive on volumetric, structural, vibrational properties, and size and shape of crystals were studied. We have revealed that ten equivalents of BA significantly enhance the porosity of obtained materials and lead to the formation of well-shaped nanoparticles. Moreover, the high concentration of BA changes the NDC/BDC ratio in the resulting MOF. In the second part of the work, using a post-synthetic exchange, we have decorated defect pores of UiO-66 type MOFs with 3-phosphonopropionic acid molecules. Using FTIR analysis and DFT calculations, we proved that 3-phosphonopropionic acid molecules bond to the $\mathrm{Zr}^{4+}$ ions by $\mathrm{PO}_{4}$-groups instead of $\mathrm{COOH}$ groups.
\end{abstract}

\section{Introduction}

Metal-organic frameworks (MOFs) are porous compounds that garnered increasing attention due to their numerous applications [1-7]. The success of MOFs in such different fields could be attributed to the high specific surface area, flexible structure, and tunable properties. One of the features of MOFs is their modular structure: they are constructed from organic and inorganic units [8-10]. Inorganic units contain clusters of metal ions with oxygen or nitrogen (with other nonmetals), these clusters are often designated as secondary building units (SBUs). Organic units are organic molecules, which are bonded to SBUs to form a 3D framework. Various combinations of these units result in numerous structures with specific properties, pore sizes, and geometry. In 2008 Lillerud and coworkers reported on the new UiO-66 family of MOFs with exceptional thermal and chemical stability [11]. SBU of UiO-66 contains six zirconium ions, each of them is coordinated by eight oxygen ions -4 from the linker's carboxylic groups and four oxygen bridge ions to other $\mathrm{Zr}^{4+}$. Each SBU is coordinated by 12 linker molecules [12]. The strong coordination bonds between the $\mathrm{Zr}^{4+}$ ions and the carboxylate ligands, along with the robust hexa-nuclear SBUs, make UiO-MOFs an exceptional family of structures, notably stable towards common solvents, moisture, heating $[11,13,14]$. 
The linker of the first member of this family - UiO-66 - is 1,4-benzene dicarboxylate (BDC). The UiO-66 is stable up to $500{ }^{\circ} \mathrm{C}$, it is quite resistant to many solvents, and to high external pressures $[15,16]$. Functional groups could be incorporated into the MOF structure to provide special functionality $[17,18]$. In our recent work, we have obtained UiO-66-NDC with 1,4-naphthalene dicarboxylate (NDC) as a linker, and a set of MOFs contained a mixture of two linkers - NDC and BDC - in one phase [19, 20]. It allowed us to tune hydrogen adsorption properties. The other option to tune properties of MOFs is "defect engineering" - the practice of manipulating defects via controlled, synthetic means. Two types of defects were reported for UiO-66: missing linker defects and missing cluster defects [21-23]. Missing-linker defect forms when the linker is removed from the structure. It generates coordination vacancies on two adjacent SBUs. When the concentration of missing-linker increases, they could join together in one region. It results in the formation of missing-cluster defects - the removal of SBU with 12 neighbor linkers. It generates one vacancy on each neighbor SBU. Vacancies, produced by missing-linker defects and missingcluster defects, could be temporarily compensated by water, chloride, hydroxide, or other [24]. The presence of defects affects such properties as catalytic activity, gas capacity, separation properties $[21,22,25]$. The most widely used technique allowed to introduce defects into the $\mathrm{UiO}$ structure is coordination modulation. According to this method, mono-carboxylic (or inorganic) acids could be added into the reaction mixture during the synthesis. The modulator effect on the crystal growth process could be described in two steps. First, modulator molecules coordinate zirconium ions, and, therefore, linker molecules could not directly interact with $\mathrm{Zr}^{4+}$. Instead of it, linkers have to substitute modulator molecules. This process results in the hindrance of the nucleation stage and deceleration of crystal growth. Finally, the modulator additive allowed to obtain big crystals with improved morphology. The high concentration of the modulator obstructs the interaction of the linker and $\mathrm{Zr}^{4+}$ ions. So, part of unsubstituted modulator molecules coordinates zirconium ions even after the formation of the crystal. As the modulator has only one carboxylic group, it could coordinate only one SBU. This leads to the formation of defects in the structure [20, 23, 26, 27]. So, varying modulator concentration, one could tune particle size and morphology, defect concentrations, the available pore volume of resulting materials. Moreover, it was reported that defects could be post-synthetically functionalized [17, 28-31]. It allowed incorporating such functional groups into the UiO-66 structure, which could not be directly used to construct MOF. Recently modification of MOFs with phosphonic acids became extremely attractive $[32,33]$. Surface modified hybrid materials have been applied in biological microarrays, drug delivery systems, immobilized homogeneous catalysts, and optoelectronics [34].

In the present work, we have investigated the effect of modulator additive on properties of MOFs with mixed linkers - NDC and BDC. Moreover, obtained materials were used for post-synthetic modification with 3-phosphonopropionic acid.

\section{Experimental part}

\subsection{Synthesis}

Starting materials: $\mathrm{ZrCl}_{4}, 1$,4-naphthalenedicarboxylic acid $\left(\mathrm{H}_{2} \mathrm{NDC}\right)$, terephthalic acid $\left(\mathrm{H}_{2} \mathrm{BDC}\right)$, benzoic acid (BA), N, N-dimethylformamide (DMF), 3-phosphonopropionic acid (PhPA) were purchased from Alfa Aesar and used without further purification. Deionized (DI) water (18 $\mathrm{M} \Omega \mathrm{cm}$ ) was obtained from a Simplicity UV ultrapure water system. In a typical procedure, $\mathrm{ZrCl}_{4}$ was dissolved in DMF under magnetic stirring, then DI water was added. After that, the respective amount of BA was added, and after complete dissolving respective amount of linkers $\left(\mathrm{H}_{2} \mathrm{NDC}\right.$ and $\mathrm{H}_{2} \mathrm{BDC}$ ) was poured to the clear solution (Table 1 , Table $\mathrm{S} 1$ ). The conical flask was capped and place into the preheated oven at $120{ }^{\circ} \mathrm{C}$ for $24 \mathrm{~h}$. After cooling to room temperature, white 
precipitates were collected using centrifugation, washed one time with DMF and one time with methanol, and dried at $60{ }^{\circ} \mathrm{C}$ overnight.

For modulator exchange experiments, $0.05 \mathrm{~g}$ of $\mathrm{MOF}$ was activated at $100{ }^{\circ} \mathrm{C}$ under the vacuum for two hours. After cooling down, powders were mixed with $5 \mathrm{ml}$ of $0.1 \mathrm{M} \mathrm{DMF}$ solution of $\mathrm{PhPA}$ and were held under magnetic stirring for $72 \mathrm{~h}$. Powders were collected by centrifugation and washed two times with methanol. Samples were designated by an additional letter "P". For example, the sample "25NDC-0-P" was obtained from sample 25NDC-0 after interaction with PhPA.

Table 1. Molar ratios of starting materials and designations of obtained samples.

\begin{tabular}{|c|c|c|c|c|c|c|}
\hline \multirow{2}{*}{$\begin{array}{l}\text { Sample } \\
\text { designation }\end{array}$} & \multicolumn{6}{|c|}{ The molar ratio of components used for the synthesis } \\
\hline & $\mathrm{ZrCl}_{4}$ & $\mathrm{H}_{2} \mathrm{NDC}$ & $\mathrm{H}_{2} \mathrm{BDC}$ & $\mathrm{H}_{2} \mathrm{O}$ & $\mathrm{BA}$ & DMF \\
\hline 25NDC-0 & \multirow{3}{*}{1} & \multirow{3}{*}{0.25} & \multirow{3}{*}{0.75} & \multirow{3}{*}{3} & 0 & \multirow{3}{*}{300} \\
\hline 25NDC-10 & & & & & 10 & \\
\hline 25NDC-60 & & & & & 60 & \\
\hline 50NDC-0 & \multirow{3}{*}{1} & \multirow{3}{*}{0.5} & \multirow{3}{*}{0.5} & \multirow{3}{*}{3} & 0 & \multirow{3}{*}{300} \\
\hline 50NDC-10 & & & & & 10 & \\
\hline $50 \mathrm{NDC}-60$ & & & & & 60 & \\
\hline UiO-66-0 & \multirow{3}{*}{1} & \multirow{3}{*}{0} & \multirow{3}{*}{1} & \multirow{3}{*}{3} & 0 & \multirow{3}{*}{300} \\
\hline UiO-66-10 & & & & & 10 & \\
\hline UiO-66-60 & & & & & 60 & \\
\hline 100NDC-0 & \multirow{3}{*}{1} & \multirow{3}{*}{1} & \multirow{3}{*}{0} & \multirow{3}{*}{3} & 0 & \multirow{3}{*}{300} \\
\hline 100NDC-10 & & & & & 10 & \\
\hline 100NDC-60 & & & & & 60 & \\
\hline
\end{tabular}

\subsection{Methods of characterization}

Powder XRD patterns were recorded on Bruker D2 PHASER X-ray diffractometer $\left(\mathrm{CuK}_{\alpha}, \lambda=\right.$ $1.5417 \AA$ ) with a step of $0.02^{\circ}$. The analysis of diffraction patterns was done in Jana2006 software [35]. Thermogravimetric analysis (TGA) was performed on the thermal gravimetric analyzer (Netzsch). Samples in corundum crucibles were heated with a rate of $10 \% \mathrm{~min}$ in the flux of air. The specific surface area (BET) values of the samples were determined from nitrogen adsorption/desorption isotherms at $-196{ }^{\circ} \mathrm{C}$ obtained on Accelerated Surface Area and Porosimetry analyzer ASAP 2020 (Micromeritics). The samples were activated at $150{ }^{\circ} \mathrm{C}$ for $12 \mathrm{~h}$ under a dynamic vacuum before the measurement. Transmission electron microscopy (TEM) was performed on the FEI Tecnai G2 Spirit TWIN transmission electron microscope operated at an accelerating voltage of $80 \mathrm{kV}$. IR spectra were measured on a Bruker Vertex 70 spectrometer in ATR geometry (Attenuated total reflectance) using an MCT detector and a Bruker Platinum ATR attachment. The spectra were measured in the range from 5000 to $30 \mathrm{~cm}^{-1}$ with a resolution of 1 $\mathrm{cm}^{-1}$ and 64 scans. The reference was air. ${ }^{1} \mathrm{H}$ NMR spectra were recorded on a Bruker AVANCE$600(600 \mathrm{MHz})$ spectrometer in $\mathrm{D}_{2} \mathrm{O}$. Prior to measurements, samples 50NDC-0 and 50NDC-60 were dissolved in deuterated alkali medium $\left(1 \mathrm{M} \mathrm{NaOH}\right.$ in $\left.\mathrm{D}_{2} \mathrm{O}\right)$, centrifugated, and separated from the solid part. All organic components after this preparation were transferred to the liquid part as corresponding sodium salts and used for NMR measurements. White precipitate contained zirconium in the form of hydrated oxide. The M4 TORNADO was used for sample characterization using small-spot micro X-ray fluorescence (Micro-XRF) analysis. 


\section{Results and discussion}

\section{1. Samples with BDC/NDC linkers}

All obtained samples had a UiO-66 structure type, according to PXRD (Figure 1). Details of profile analysis are provided in SI Section 3. Lattice constants of the mixed-linker samples had the prevailing trend - for each BA concentration lattice parameter $a$ increased with the increase of NDC content (Table 2, Fig. S3 in SI)). It could be attributed to the stress created by extra benzene rings of the NDC linker in comparison with the BDC ligands in good agreement with previously reported data [19]. The additive of 60 eq. of BA decreased the yield of reaction almost twice, while ten eq. of BA did not affect the amount of the product, and after $24 \mathrm{~h}$, the yield was near $100 \%$.
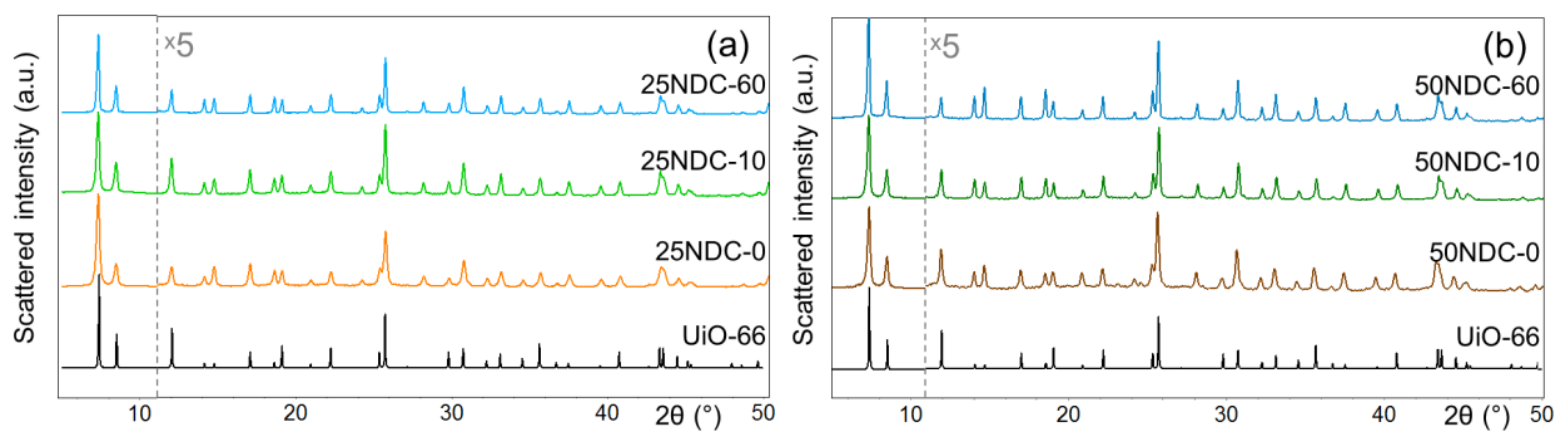

Figure 1. PXRD profiles of synthesized samples with mixed linkers and various BA additives. The black profile corresponds to the UiO-66 structure. It was calculated according to the data [12]. Patterns are shifted along the y-axis. Intensities are multiplied by five for 20-region after dotted lines. Manipulations were applied for better representation.

Table 2. Some properties of obtained samples. Theoretical molar weights were calculated according to compositions of activated samples (for details, see SI Table S2).

\begin{tabular}{|c|c|c|c|c|c|c|}
\hline \multirow{3}{*}{$\begin{array}{c}\text { Sample } \\
\text { designation }\end{array}$} & \multirow{3}{*}{$a, \AA$} & \multirow{3}{*}{$\begin{array}{c}\text { SSA } \\
(\mathrm{BET}), \\
\mathrm{m}^{2} / \mathrm{g}\end{array}$} & \multirow{3}{*}{$\begin{array}{c}\text { Pore } \\
\text { volume, } \\
\mathrm{m}^{3} / \mathrm{g}\end{array}$} & \multicolumn{3}{|c|}{ TGA } \\
\hline & & & & \multicolumn{2}{|c|}{ Molar weight, $\mathrm{g} / \mathrm{mol}$} & \multirow{2}{*}{$\begin{array}{c}\text { Missing } \\
\text { linkers, \% }\end{array}$} \\
\hline & & & & theoretical & experimental & \\
\hline 25NDC-0 & $20.7524(9)$ & 1029 & 0.41 & 1703.2 & 1664.1 & 4.1 \\
\hline 25NDC-10 & $20.7688(7)$ & 1272 & 0.61 & 1703.2 & 1700.2 & 0.3 \\
\hline 25NDC-60 & $20.7630(8)$ & 1319 & 0.52 & 1703.2 & 1581.9 & 12.6 \\
\hline 50NDC-0 & $20.7719(9)$ & 787 & 0.34 & 1778.3 & 1685.4 & 8.9 \\
\hline 50NDC-10 & $20.7799(8)$ & 948 & 0.43 & 1778.3 & 1729.7 & 0.4 \\
\hline 50NDC-60 & $20.7896(7)$ & 1195 & 0.48 & 1778.3 & 1707.6 & 6.8 \\
\hline UiO-66 & $20.7330(10)$ & 1061 & 0.47 & 1628.1 & 1508.3 & 7.4 \\
\hline 100NDC & $20.8279(16)$ & 488 & 0.26 & 1928.4 & 1877.9 & 2.6 \\
\hline
\end{tabular}

TGA curves are presented in Figure 2 by rescaling the weight at the end of the process to $100 \%$ and attributing it to the pure $\mathrm{ZrO}_{2}$ phase. The theoretical plateau was calculated according to the molar weight of ideal defect-free 25-NDC and 50NDC samples. First weight loss at a temperature less than $90^{\circ} \mathrm{C}$ associated with water desorption, while the next step at $290^{\circ} \mathrm{C}$ could be attributed to DMF evacuation from the pores. The temperature of linker decomposition and structure collapse is about $450^{\circ} \mathrm{C}$. DSC curves measured in airflow for both samples exhibit peak located at about $500{ }^{\circ} \mathrm{C}$, which corresponded to the exothermic process of framework decomposition. Experimental 
weight losses of all samples are lower than theoretically calculated according to the ideal structure. UiO-66 type MOFs are incredibly tolerant of a high concentration of defects. Moreover, it was reported that BA additives initiate the formation of defects in UiO-66 and UiO-66-NDC structures $[20,22,23,36]$. Concerning all these points, the presence of defects in the obtained structures is rather an expected result. However, it was not trivial to evaluate the quantities of defects. According to previous reports, an increase of BA concentration in the reaction mixture results in more defective UiO-66 and UiO-66-NDC structures [20, 23]. Using two linkers and modulator in one reaction bath leads to more complicated trends. In fact, the samples with the ideal non-defect structure were obtained with ten equivalents of BA - samples 25NDC-10 and 50NDC-10. Samples obtained without any modulator or with 60 equivalents of BA had lower weight loss in comparison with the ideal structure. According to the method suggested by Valenzano et al. in 2011, the magnitude of the decomposition weight loss step could indicate the number of missing linkers [12]. In this way, the samples 25-NDC-0 and 50-NDC-0 have weight losses upon combustion that correspond to $4.1 \%$ and $8.9 \%$ missing linkers, respectively. However, moving to the samples 25NDC-60 and 50NDC-60, we should consider that during the crystallization process, three acids compete for coordination of $\mathrm{Zr}^{4+}$ ions $-\mathrm{H}_{2} \mathrm{BDC}, \mathrm{H}_{2} \mathrm{NDC}$, and $\mathrm{BA}$. Therefore, the ratio between $\mathrm{BDC} / \mathrm{NDC}$ in the final product could deviate from the stoichiometric one. Particularly, a higher concentration of NDC linker could partially compensate defects effect on weight loss. In this way, we could not make conclusions about the concentration of defects according to TGA data only.
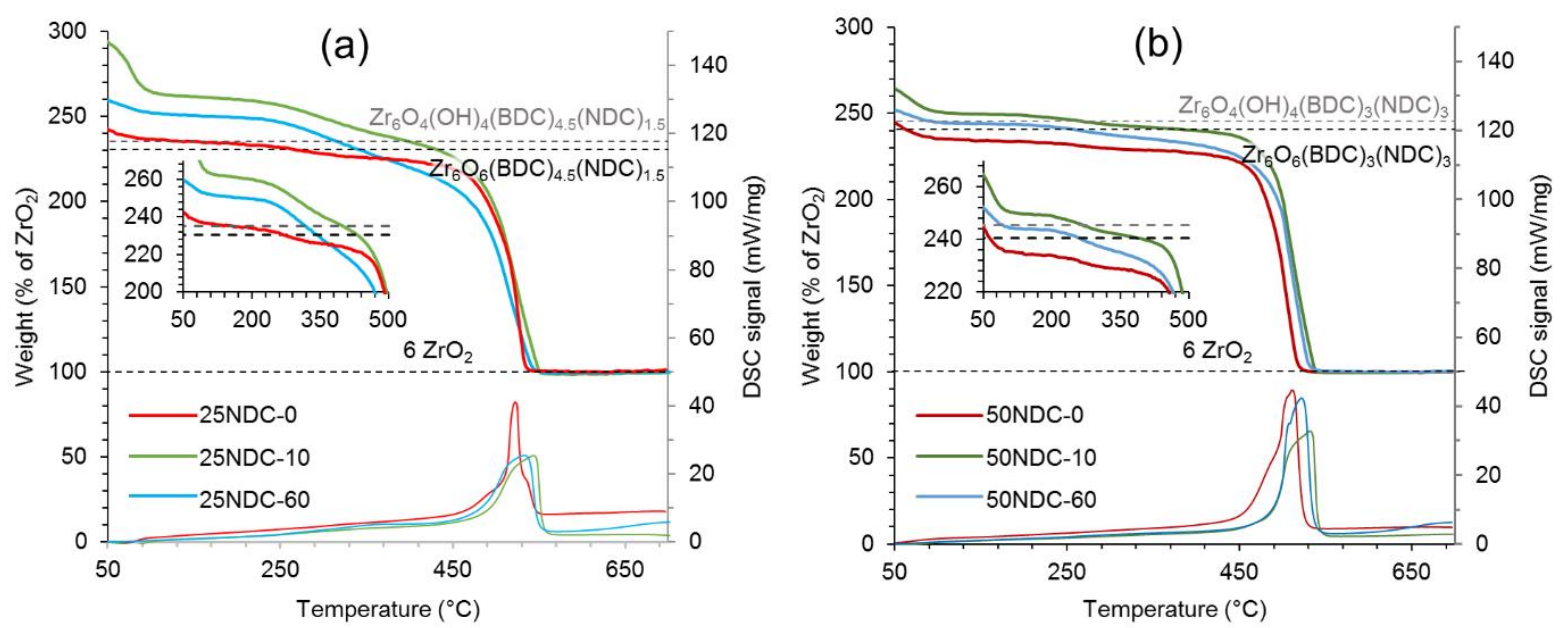

Figure 2. TGA and DSC curves of synthesized samples 25NDC-0, 25NDC-10, 25NDC-60 (a) and 50NDC-0, 50NDC-10, 50NDC-60 (b). TGA plots were renormalized, referring to the remaining weight at high temperature $\left(\mathrm{ZrO}_{2}\right)$ as $100 \%$. Gray dotted lines represent the normalized weight of the hydrated sample, while black line - dehydrated one.

Nitrogen adsorption/desorption isotherms are provided in Figure 3. All of them could be attributed to Type I (IUPAC classification), which are typical for microporous materials. Adsorption plateau, as well as SSA values, are strongly correlated with the BDC/NDC ratio in the sample and BA additive used in the synthesis. Higher NDC content leads to a lower SSA because extra benzene rings of NDC linker occupy part of the pore volume. SSAs of samples with molar ratio NDC/BDC 50/50 (50NDC-0, 50NDC-10 and 50NDC-60) and 100/0 (100NDC-0, 100NDC-10 and 100NDC60 ) increase smoothly with increasing modulator concentration in good agreement with previously reported data [20, 23]. However, samples obtained with ten equivalent of BA (UiO-66-10, 25NDC-10, 50NDC-10, and 100NDC-10) exhibit two new trends: hysteresis loop in the region near $\mathrm{P} / \mathrm{P}_{0}=1$ and higher SSA than it was expected. Substitution of BDC linker with NDC one somehow smooth the last trend, while the hysteresis loop could be observed for all compositions. 

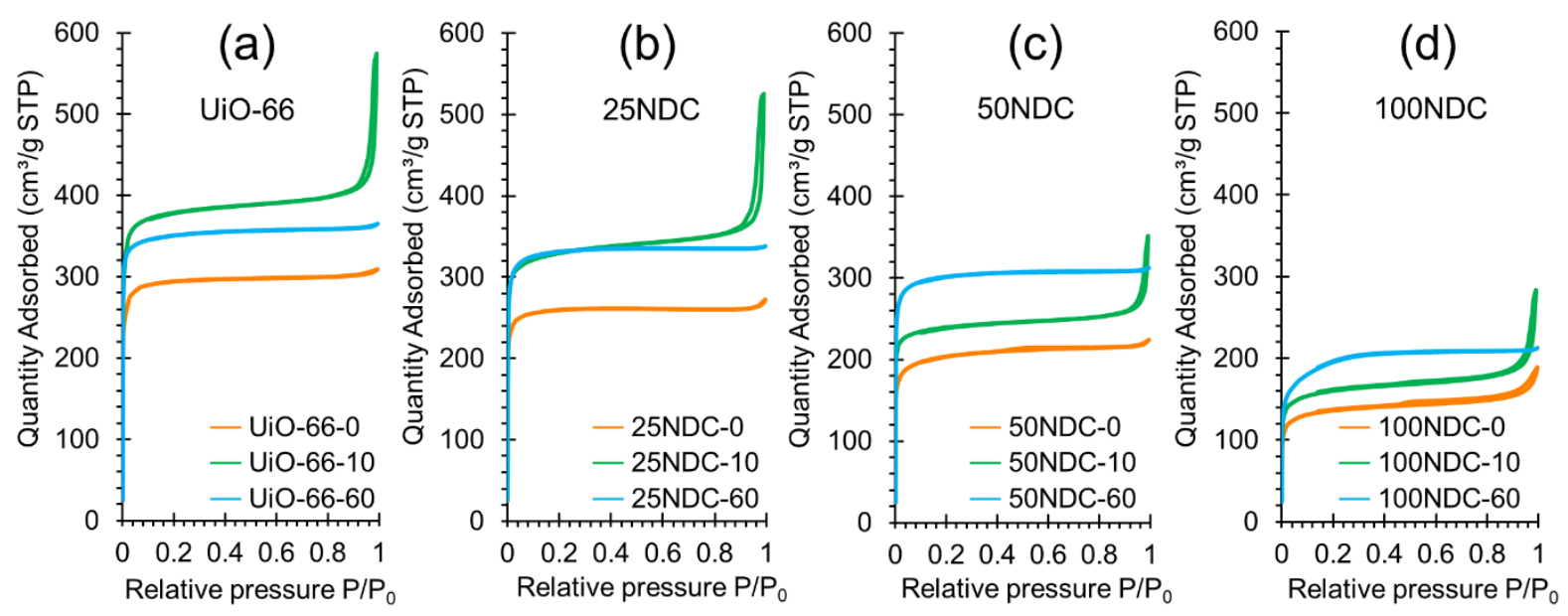

Figure 3. $\mathrm{N}_{2}$ adsorption/desorption isotherms of samples $\mathrm{UiO}-66-0, \mathrm{UiO}-66-10$ and $\mathrm{UiO}-66-60$ (a), 25NDC-0, 25NDC-10 and 25NDC-60 (b), 50NDC-0, 50NDC-10 and 50NDC-60 (c), and 100NDC-0, 100NDC-10 and 100NDC-60 (d).

Hysteresis loop on $\mathrm{N}_{2}$ adsorption/desorption isotherms of microporous materials could be attributed to adsorption in cavities between the crystallites of material. This result is in good agreement with TEM images of obtained samples: 25NDC-10 and 50NDC-10 are uniform wellshaped cubic crystals about $100 \mathrm{~nm}$, which could form a stable colloid solution in isopropanol. While samples 25NDC-0 and 50NDC-0, obtained without BA, formed aggregates composed from small crystals with different shapes and sizes. The dense packing of agglomerates does not leave any significant volume between the crystallites. Samples 25NDC-60 and 50NDC-60 contain big hexagonal crystals about 700-800 nm. It should be noted that hexagonal shape can be obtained by observing a cube along the 111 direction.
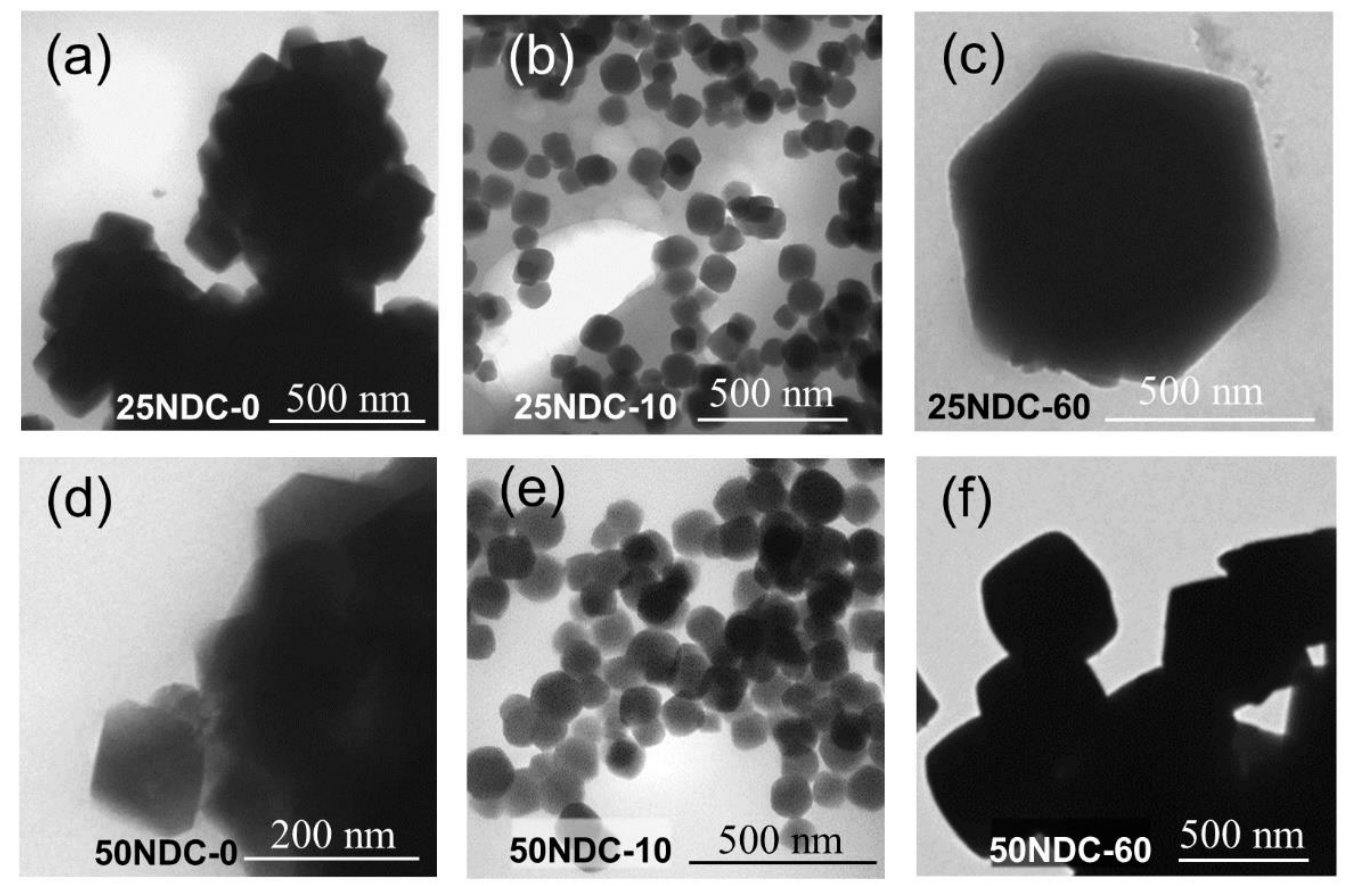

Figure 4. Representative TEM images for 25NDC-0 (a), 25NDC-10 (b), 25NDC-60 (c) and 50NDC-0 (d), 50NDC-10 (e), 50NDC-60 (f). 
FTIR spectra of all the samples are provided in Figure 5. The IR spectra are very similar in all the samples as expected, as the used linkers contain the same functional groups. However, a list of modes could be used to identify BDC or NDC linkers. Samples UiO-66-0, UiO-66-10 and UiO66-60 with only BDC linker contain characteristic bands at 550, 740, 1390 and $1505 \mathrm{~cm}^{-1}$. Samples 100NDC-0, 100NDC-10, and 100NDC-60 with only NDC linker show the following list of specific vibrations: $675,790,1210,1265,1365,1410$, and $1460 \mathrm{~cm}^{-1}$. A band at $740 \mathrm{~cm}^{-1}$ could be assigned to $\mathrm{C}-\mathrm{H}$ wagging in-plane vibrations of the BDC linker, while the naphthalene ring in NDC linker shifts it to $790 \mathrm{~cm}^{-1}$. Absorption at $1390 \mathrm{~cm}^{-1}$ is attributed to the O-C-O symmetric stretching in a carboxylic group of BDC linker, while O-C-O group of NDC linker has the same band at $1410 \mathrm{~cm}^{-1}$. Two peaks at 1365 and $1460 \mathrm{~cm}^{-1}$ could be assigned to $\mathrm{C}-\mathrm{C}$ stretching and $\mathrm{C}-\mathrm{H}$ bending vibrations of the NDC linker. Samples 50NDC and 25NDC with mixed linkers contain bands characteristic for both NDC and BDC molecules. BA additive does not modify spectra significantly; however, some changes are observed. First of all, new bands (at 717, 938, and $1025 \mathrm{~cm}^{-1}$ ) appear in the spectra of samples with BA additive. They could be assigned to BA molecules, which are coordinate $\mathrm{Zr}^{4+}$ ions in the defect pores. Relative intensities of these peaks increase in good agreement with the increase of BA concentration used for the synthesis. We have compared FTIR spectra of 25NDC and 50NDC samples with a mechanical mixture of samples UiO-66-0 and 100NDC-0 in the molar ratio 75:25 and 50:50, respectively. Samples 25NDC-0, 25NDC-10, 50NDC-0, and 50NDC-10 coincide well with the spectra of corresponding mechanical mixtures. However, the growth of BA concentration leads to an increase in relative intensities of NDC modes (Figure 5b). It could indicate that the NDC/BDC ratio in the synthesized material slightly deviates from the theoretically predicted according to weights used for synthesis. In fact, BDC content in the samples 50NDC-60 and 25NDC-60 is lower than in 50NDC-0 and 25NDC-0, respectively.
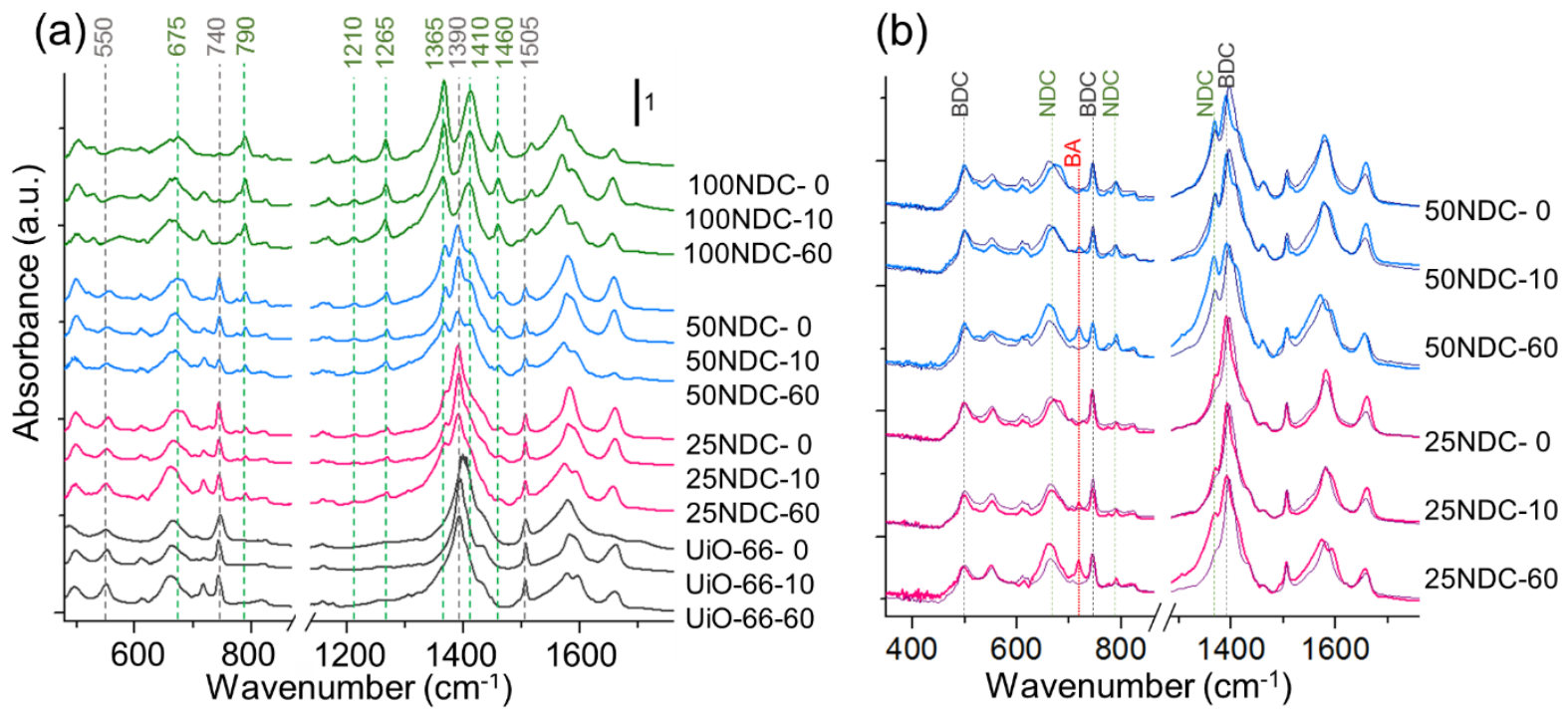

Figure 5. FTIR spectra of all obtained samples (a) and magnified regions $350-850 \mathrm{~cm}^{-1}$ and 1300-1750 cm-1 for 25NDC-0, 25NDC-10, 25NDC-60, 50NDC-0, 50NDC-10 and 50NDC-60 samples $(b)$. Thin lines in part (b) represent spectra of mechanical mixture UiO-66 with 100NDC in molar ratio 75:25 (pink lines) and 50:50 (blue lines).

As it was reported previously, the NMR technique could be applied to MOFs to identify and quantify the organic components [22, 23]. The fragments of the obtained spectra are presented in Figure 6. The ${ }^{1} \mathrm{H}$ NMR spectrum of sample 50NDC-0 exhibits signals that correspond to all protons of BDC and NDC molecules, which are indicated by Latin letters in Figure 6a. Thus, the 
protons of the BDC, due to their complete magnetic equivalence, appear as a singlet signal at 7.75 ppm. Protons D of NDC appears as a singlet signal at $7.45 \mathrm{ppm}$, while protons B and C appear as multiplet signals at 7.50 and $8.09 \mathrm{ppm}$. It should be noted that in BDC, there are four hydrogen nuclei of the same type (A), and in the NDC, there are two protons of three types (B, C, and D). Taking the integral intensity of the signal of the BDC protons equal to four, it was found out that the signals of the protons of the NDC possess two-proton intensity, i.e., the ratio of compounds in solution is $1: 1$.

The spectrum also contains DMF signals, namely, the signal of the formyl group proton at 8.33 ppm and $\mathrm{N}-\mathrm{CH}_{3}$ groups at $2.13 \mathrm{ppm}$. The position and shape of these signals, as well as the ratio of their integral intensities, equal to 1: 6 , fully confirm this assumption. In this case, about 0.91 DMF molecules per one molecule of BDC.

The ${ }^{1} \mathrm{H}$ NMR spectrum of sample 50NDC-60 is considerably more complicated in comparison with the spectrum of 50NDC-0. Still, it also shows signals of various magnetically nonequivalent protons, which are indicated by Latin letters in Figure 6b. Thus, proton $\mathrm{G}$ of BA appears as a triplet at $7.42 \mathrm{ppm}$, protons $\mathrm{F}$ as a triplet with doubled integral intensity at $7.35 \mathrm{ppm}$, and protons $\mathrm{E}$ as a doublet that merges with the signal of BDC protons and form a multiplet at about $7.73-7.75 \mathrm{ppm}$. The protons of the NDC, as in the previous case, appear as two characteristic multiplets and one singlet with the same integral intensity ( $\sim 1.87$ relative to the weighted average proton of BA). An analysis of the integral intensities of all signals allowed us to establish the ratio of NDC:BDC:BA as 1:0.91:1.1 (in mole percent). DMF signals occur at 8.33 and $2.12 \mathrm{ppm}$. (ESI), with about 0.57 molecules of DMF per one molecule of BDC.

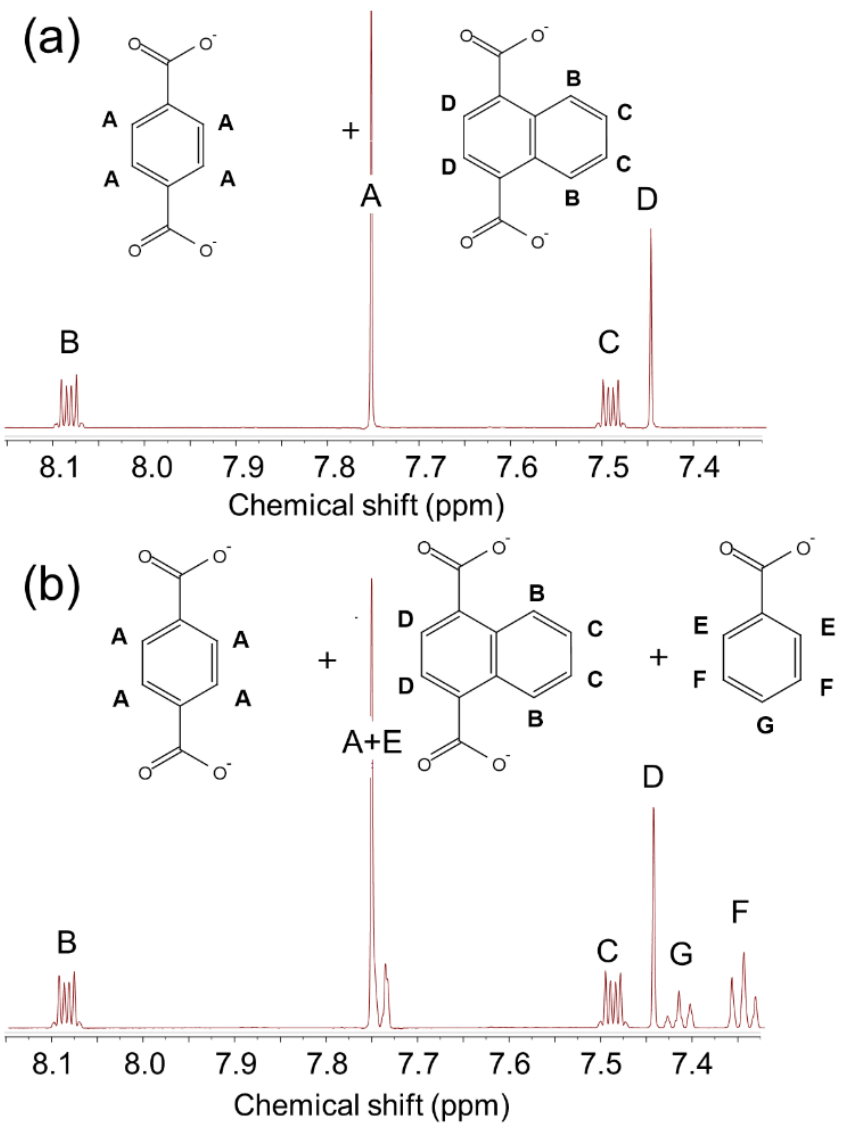

Figure 6. Fragments of the ${ }^{1} H$ NMR spectra in the region of aromatic protons of the sample 50NDC (a) and 50NDC-60 (b). 
Summarizing all observed experimental data, we could propose the following process. Without modulator, the direct interaction of zirconium salt with linkers produces a lot of nuclei. As it was reported, UiO-66 nuclei have structure close to tetragonal zirconium oxide [37]. These nuclei join together and form agglomerates instead of individual crystal growth in good agreement with TEM images (Figure 4 a,d). It results in imperfections in the crystal structure and could block pores. Lower experimental weight loss could be attributed to the high concentration of nuclei with higher zirconium content. Imperfections in the crystals and aggregation result in blocked pores, and therefore, lower available pore volume. Modulator additive affects the crystallization process. Benzoic acid and linker molecules in reaction mixture compete for zirconium coordination. BA concentration in the reaction mixture is 10/60 times higher than the linker ones. So, the first coordination sphere forms from monodentate modulator molecules. However, interaction with the bidentate linker is an energetically preferable process. During crystal growth, BA will be substituted with linkers. BA-sphere around zirconium ions obstructs the nucleation stage and slow down crystal growth. Not all BA molecules will be substituted, some of them will coordinate zirconium in the final crystal, and it will lead to the formation of defects. We suppose, that 10 eq. of BA leads to the formation of missing-linker defects, while 60 eq. of BA increase defect concentration, and cooperated local defects form missing-SBU defects. This process was reported for UiO-66 and UiO-66-NDC [20]. Missing-SBUs lead to high porosity and low linker content in good agreement with TGA data. Missing linkers do not significantly affect experimental weight loss in our case when benzoate ions coordinate zirconium in defect pores. While specific surface areas of samples obtained with 10 of BA are significantly higher than for respected samples obtained without a modulator. We also observed that 60 eq. of BA not only initiate the formation of missing-SBU defects but additionally affect the ratio between NDC and BDC linkers. Namely, samples 50NDC-60 and 25NDC-60 exhibited lower BDC content than respective samples obtained without modulator or with 10 eq. of BA. This effect was not reported previously. We suppose that it could be assigned to interaction between naphthalene rings of NDC linkers and slow crystal growth. In this case, two naphthalene rings of NDC linkers provide more available delocalized $\pi$-electrons for pi staking intermolecular interaction then BDC linkers. And it could increase the local concentration of $\mathrm{H}_{2} \mathrm{NDC}$ molecules in the solution near nuclei coordinated with NDC linkers.

\subsection{Post-synthetic exchange}

After post-synthetic exchange experiments, all samples preserved high crystallinity according to XRD analysis (Figure 7a). Phosphorus concentration in the samples functionalized with PhPA molecules was determined using XRF analysis (Figure 7b). It was observed that both the BDC/NDC ratio and BA concentration affect phosphorus content in MOF. Samples obtained without BA additive did not contain a significant amount of phosphorus. $\mathrm{P}$ content in samples 25NDC, 50NDC, and UiO-66 are in good agreement with SSA values. Namely, 25NDC-10 and UiO-66-10 had higher SSA than 25NDC-60 and UiO-66-60, so the highest P concentrations are attributed to samples 25NDC-10 and UiO-66-10. Sample 50NDC-60 had higher SSA than 50NDC-10 and, consequently, higher phosphorus content. The second trend observed in this experiment: substitution of BDC linker with NDC one obstructs post-synthetic exchange. So, the increase of NDC content decreases the concentration of phosphorus in the final material. We suppose that additional benzene rings in the NDC linker reduced windows to the pores and pore volume, and it obstructs interaction with PhPA. 

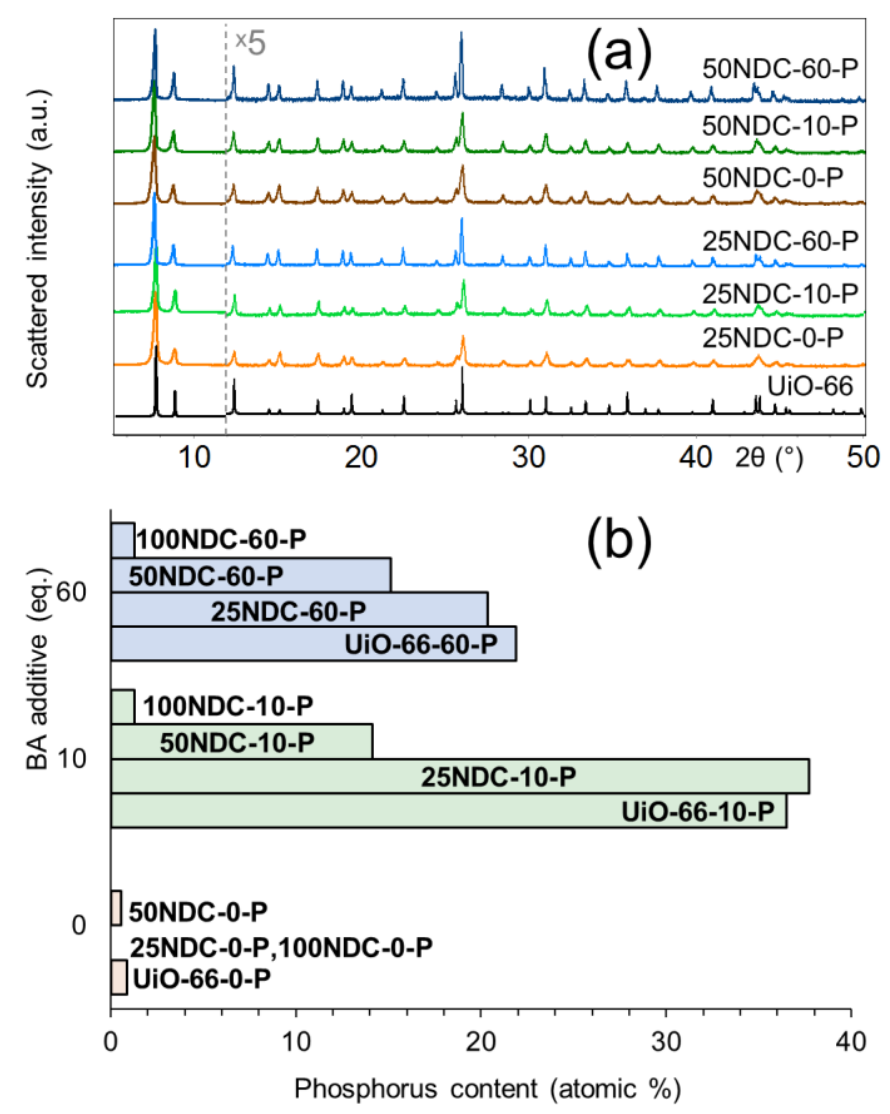

Figure 7. (a) XRD profiles of samples after interaction with PhPA. The black profile corresponds to the UiO-66 structure [12]. (b) Phosphorus content in each sample according to XRF analysis.

For the samples 25NDC-0-P, 25NDC-10-P, and 25NDC-60P nitrogen adsorption isotherms were measured (Figure 8), and SSAs were calculated according to the BET model. SSA of the sample 25NDC-0 almost did not reduce after interaction with PhPA (25NDC-0 $1029 \mathrm{~m} / \mathrm{g} ; 25 \mathrm{NDC}-0-\mathrm{P}$ $1015 \mathrm{~m}^{2} / \mathrm{g}$ ) in good agreement with XRF data, which did not reveal any phosphorus in the sample 25NDC-0-P. Sample 25NDC-10-P has SSA in 1.7 times lower than the initial 25NDC-10 (755 $\mathrm{m}^{2} / \mathrm{g}$ ), while sample 25NDC-60-P reduced SSA in 1.3 times $\left(1011 \mathrm{~m}^{2} / \mathrm{g}\right.$ ) (Table 2).
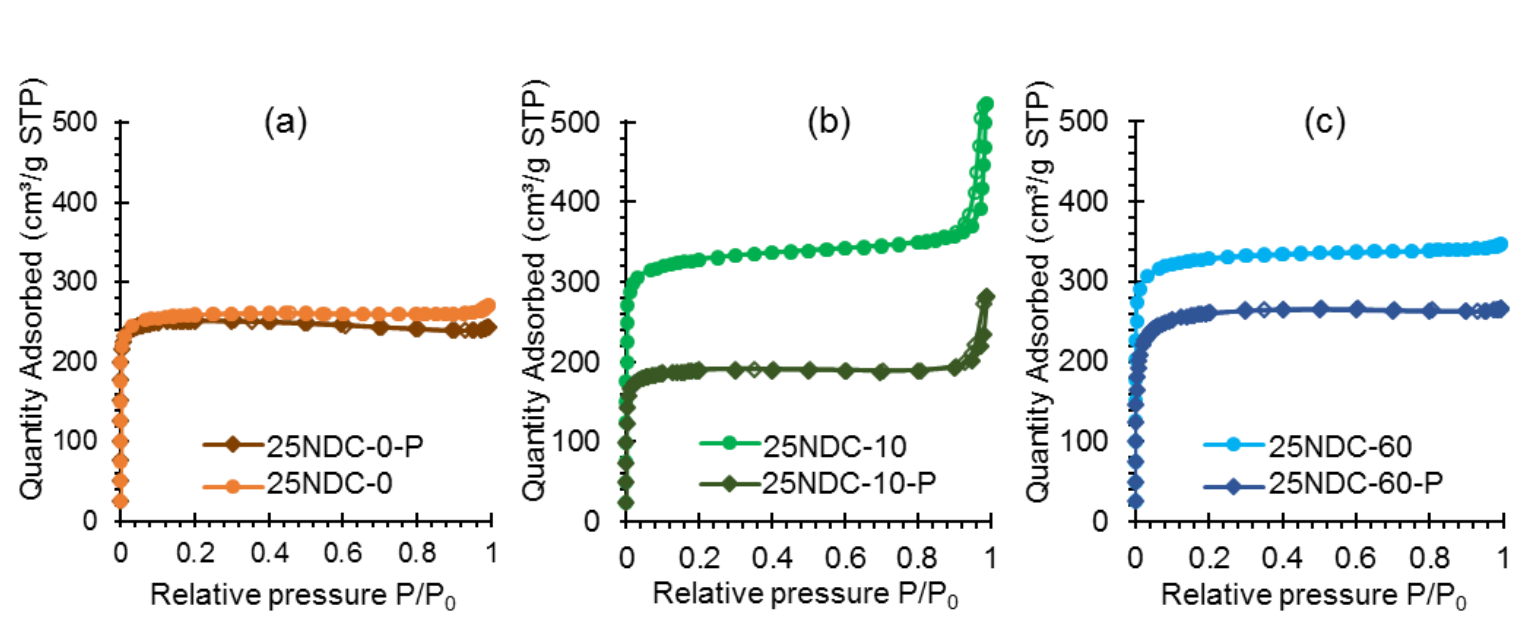

Figure 8. $\mathrm{N}_{2}$ adsorption-desorption isotherms of samples 25NDC-0 and 25NDC-0-P (a), 25NDC10 and 25NDC-10-P (b), 25NDC-60 and 25NDC-60-P (c). Adsorption branches of isotherms 
contain filled markers, while desorption ones - empty markers. Round markers represent isotherms of initial samples, while diamond markers were used for samples after PhPA exchange.

The same trends could be observed from FTIR spectra analysis. Samples 100NDC-10 and 100NDC-60 did not contain a significant amount of $\mathrm{P}$ after the post-synthetic exchange, according to XRF analysis. In good agreement with these results, the FTIR spectra of samples 100NDC-10$\mathrm{P}$ and 100NDC-60-P did not reveal any modes of $\mathrm{PO}_{4}$-group (Figure 9). On the other hand, FTIR spectra of samples 50NDC-10/60-P, 25NDC-10/60-P, and UiO-66-10/60-P demonstrate new broad modes at 1035,645 and $530 \mathrm{~cm}^{-1}$ assigned to vibrations of the $\mathrm{P}-\mathrm{O}$ bond in the phosphorus tetrahedrons $[38,39]$. These modes are more pronounced in samples 25NDC-10/60-P and UiO66-10/60-P than in 50NDC-10/60-P ones in good agreement with a higher concentration of $\mathrm{P}$ determined by XRF analysis. Moreover, as it was reported previously, zirconium could form strong bonds with $\mathrm{PO}_{4}$-groups [34, 40]. In this way, PhPA should bond to the $\mathrm{Zr}^{4+}$ ion in defect pore by $\mathrm{PO}_{4}$-group instead of $\mathrm{COOH}$-group [41, 42]. This hypothesis is in good agreement with changes in the spectra of samples after modulator exchange. First of all, modes assigned to BA vibrations have vanished in the spectra of all samples except the one with NDC linker - 100NDC10 and 100NDC-60. It proves that the BA modulator was substituted with PhPA molecules. Secondly, new bands can be observed at 1690 and $1295 \mathrm{~cm}^{-1}$. The intensity of these bands decreases with the increase of NDC content: UiO-66-10-P and UiO-66-60-P samples show the most pronounced bands, spectra of 25NDC-10-P and 25NDC-60-P samples contain the same peaks with reduced intensity, in spectra of samples 50NDC-10-P and 50NDC-60-P these bands can be still distinguished, while in spectra of samples 100NDC-10-P and 100NDC-60-P these bands are not observed. Absorption bands at $1690 \mathrm{~cm}^{-1}$ correspond to a carbonyl stretch $(\mathrm{vC}=\mathrm{O})$ of the protonated carboxylic group, while deprotonated one shifts to lower energy at $1660 \mathrm{~cm}^{-1}$. $\mathrm{C}-\mathrm{OH}$ vibrations give rise to a feature $(\mathrm{vC}-\mathrm{OH})$ at $1295 \mathrm{~cm}^{-1}$, while the deprotonation of this group shifts the peak to higher energy at $1398 \mathrm{~cm}-1$ for BDC linker and $1415 \mathrm{~cm}^{-1}$ for NDC one [12, 19, 43, 44]. Deprotonated carboxylic groups comprise all obtained MOFs as a part of linkers, while protonated one could be attributed only to PhPA molecules bonded to $\mathrm{Zr}^{4+}$ with $\mathrm{PO}_{4}$-group.
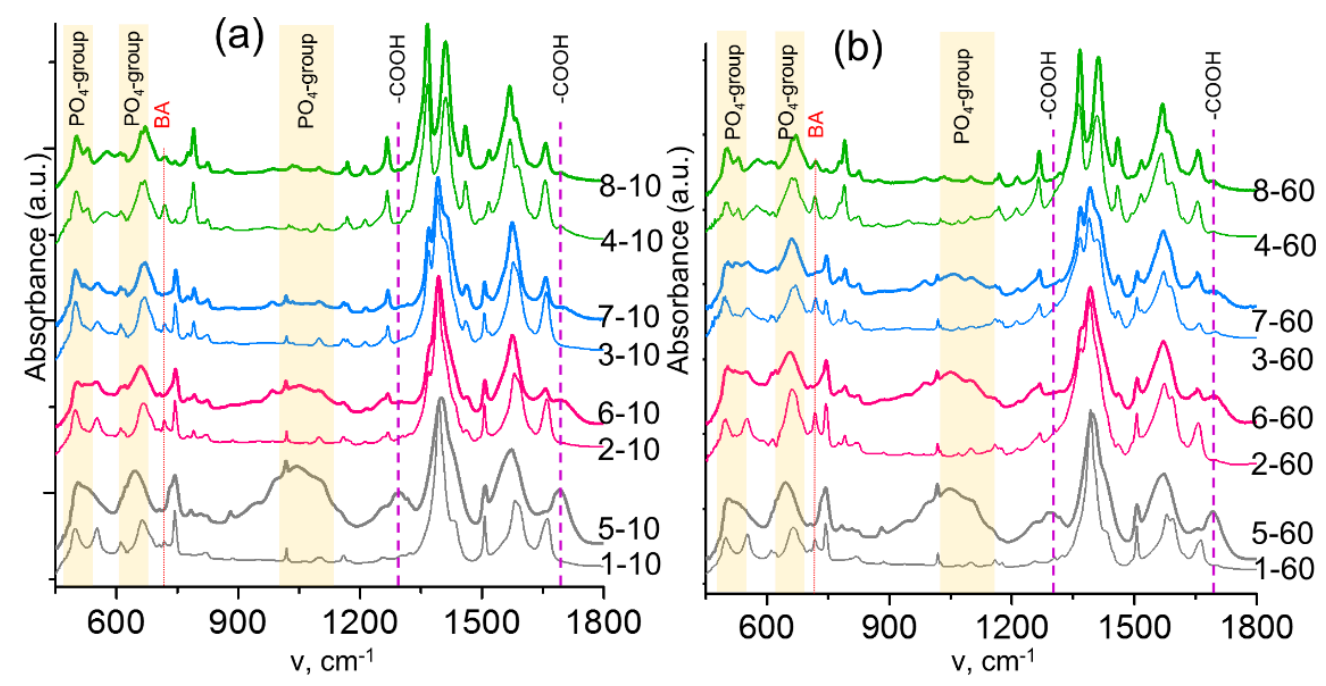

Figure 9. FTIR spectra of samples before interaction with PhPA (thin lines) and after it (bold lines). Part (a) represents spectra of samples obtained with 10 eq of BA: 1-10 - UiO-66-10, 2-10 - 25NDC-10, 3-10 - 50NDC-10, 4-10 - 100NDC-10; 5-10 - UiO-66-10-P, 6-10-25NDC-10-P, 7-10 - 50NDC-10-P, 8-10-100NDC-10-P. Part (b) represents spectra of samples obtained with 60 eq of BA: 1-60 - UiO-66-60, 2-60 - 25NDC-60, 3-60 - 50NDC-60, 4-60 - 100NDC-60; 5-60 - UiO-66-60-P, 6-60 - 25NDC-60-P, 7-60 - 50NDC-60-P, 8-60-100NDC-60-P. 


\subsection{DFT calculations}

Theoretical calculations were applied to prove our experimental conclusions. As it was mentioned above, PhPA could alternatively bond to SBU through $\mathrm{COOH}$ - or $\mathrm{H}_{2} \mathrm{PO}_{3}$ - group. For modeling of both variants, we performed density functional theory (DFT) quantum chemical calculations using Gaussian16 [45] program package in the approximation B3LYP/6-311++G(d,p) [46] with the inclusion of the dispersion correction D3BJ [47]. SBU $\mathrm{Zr}_{6} \mathrm{O}_{4}(\mathrm{OH})_{4}$ coordinated by benzoate ions instead of BDC linkers was used as the model cluster.

In the first step, we have considered two possible ways of PhPA deprotonation. The first option includes the removal of one $\mathrm{H}^{+}$from the phosphorous end of PhPA with the formation of $\mathrm{HPO}_{3}$ anion (Figure 10c). The second option is the deprotonation of the carboxylic group, which results in $\mathrm{COO}^{-}$anion (Figure 10b). Calculations showed that the deprotonated $\mathrm{PhPA}$ molecule with $\mathrm{HPO}_{3}{ }^{-}$group is $9.9 \mathrm{kcal} / \mathrm{mol}$ more stable than anion formed by deprotonation of the carboxylic group. Therefore, according to the results obtained, we could conclude that the coordination of the $\mathrm{PhPA}$ molecule to $\mathrm{SBU}$ through the $\mathrm{HPO}_{3}{ }^{-}$group is preferable.

(a)

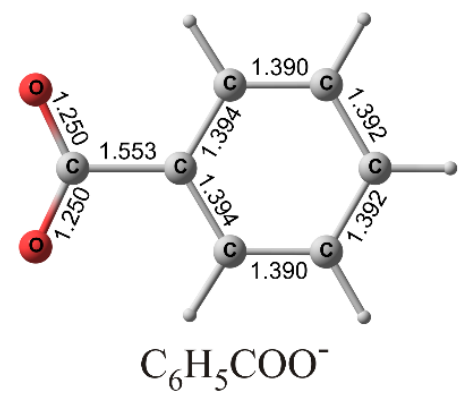

(b)

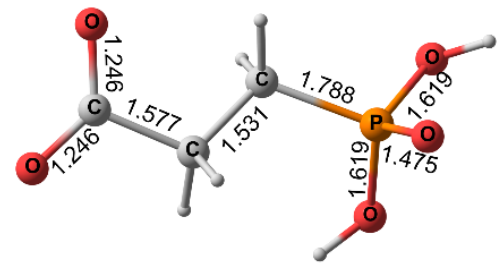

$(\mathrm{OH})_{2}(\mathrm{O}=) \mathrm{P}_{-} \mathrm{C}_{2} \mathrm{H}_{4}-\mathrm{COO}^{-}$ (c)

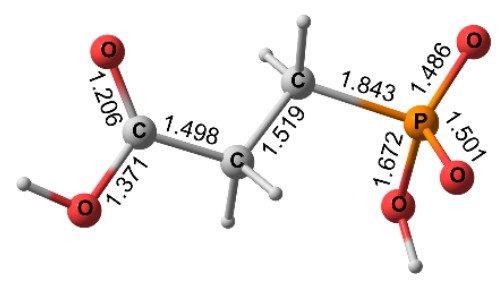

$\left(\mathrm{O}^{-}\right)(\mathrm{OH})(\mathrm{O}=) \mathrm{P}-\mathrm{C}_{2} \mathrm{H}_{4}-\mathrm{COOH}$

Figure 10. Optimized structures of isolated anions, bond lengths are given in $\AA$. Part (a) represents benzoate anion, which was considered as the coordination sphere of SBU. Parts $(b)$ and (c) show two ways of PhPA deprotonation: with the formation of $-\mathrm{COO}^{-}$anion $(b)$ and $\mathrm{H}_{2} \mathrm{PO}_{3}^{-}$anion (c).

Figure 11 shows the optimized geometries of model clusters. Structure 1, in which the benzoic acid anion represents all bridging ligands, is characterized by symmetry close to $C_{3}$. The calculated $\mathrm{Zr}-\mathrm{O}$ bond lengths are $2.24 \AA$, and the $\mathrm{Zr}-\mathrm{Zr}$ distances are $3.54 \AA$.

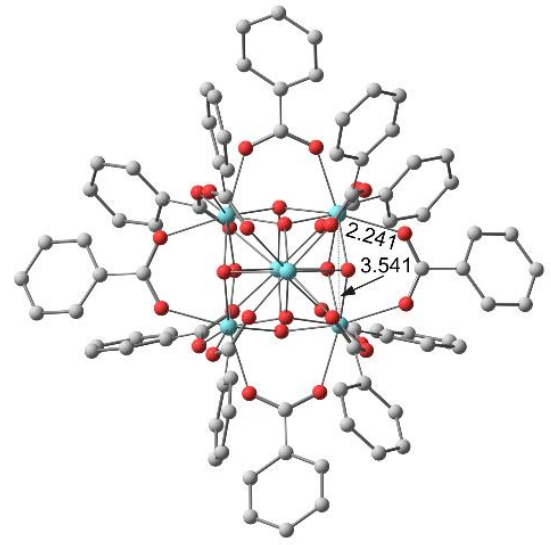

1

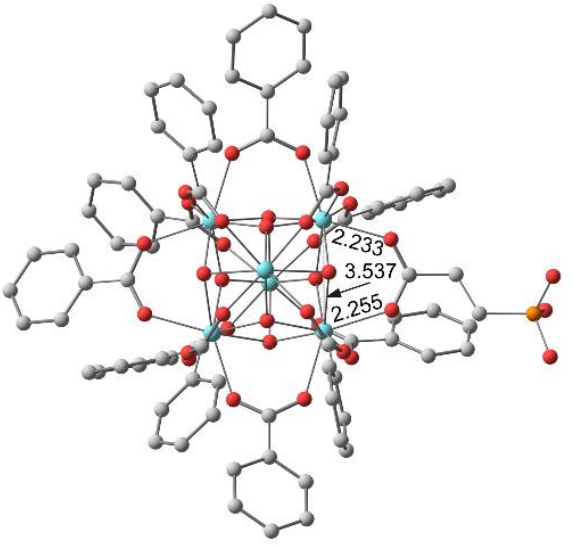

2

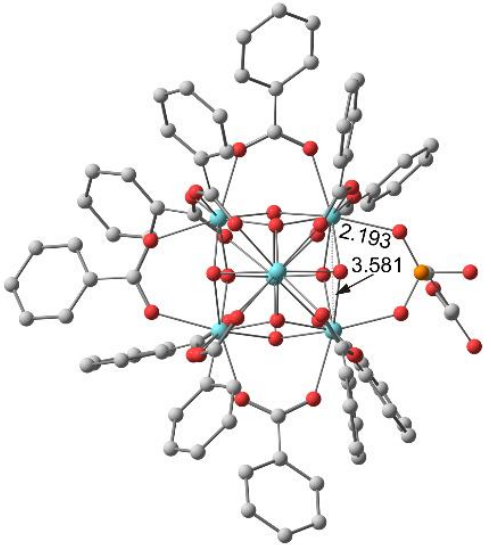

3

Figure 11. Cluster $\mathrm{Zr}_{6} \mathrm{O}_{4}(\mathrm{OH})_{4}$ coordinated by benzoate ions used for DFT optimization (1). Model structures of this cluster with one benzoate ion replaced by a PhPA molecule bonded to zirconium through $\mathrm{COOH}$ - group (2) and $\mathrm{H}_{2} \mathrm{PO}_{3}$-group (3). Hydrogen atoms are omitted for clarity; bond lengths are given in $\AA$. 
Since the PhPA molecule contains two donor centers that could form bonds with zirconium in SBU, two isomers that differ in the way of coordination were calculated. Replacing the benzoic acid anion with PhPA coordinated by the carboxylic group does not lead to a significant distortion of the framework of structure 2. The $\mathrm{Zr}-\mathrm{Zr}$ distance remains unchanged, the $\mathrm{Zr}-\mathrm{O}$ bonds change by $0.01 \AA$. At the same time, in structure 3 , characterized by the coordination of PhPA by a phosphorus group, noticeable distortions in the geometry of the coordination polyhedron are predicted. The predicted shortening of the $\mathrm{Zr}-\mathrm{O}$ bond lengths to $2.22 \AA$ and an increase in the $\mathrm{Zr}$ - Zr distance to $3.59 \AA$ lead to a decrease in the symmetry of the system. A comparison of the energy characteristics of isomers 2 and 3 indicates the preference of the latter at $19.1 \mathrm{kcal} / \mathrm{mol}$. It allows us to expect the coordination of PhPA to UiO-66 by means of the phosphorus group.

Next, we evaluated the thermodynamic stability of isomers 2 and 3 in comparison with the initial model structure 1, including the benzoic acid anion. According to calculations in the chosen approximation (B3LYP/6-311++G(d,p), D3BJ), replacing the benzoic acid anion with $\mathrm{PhPA}$ through the carboxyl group will increase the system energy by $1.8 \mathrm{kcal} / \mathrm{mol}$, which makes this process unlikely. Moreover, coordination with a phosphorus group (structure 3) is accompanied by a gain in energy $\left(E_{\text {stab }}=7.5 \mathrm{kcal} / \mathrm{mol}\right)$, which indicates a high probability of this method of PhPA introduction to UiO-66.

Thus, the DFT calculations of model fragments of MOF UiO-66 showed that from a thermodynamic point of view, the replacement of the benzoic acid anion with PhPA could only be realized if the last phosphoric group is coordinated.

\section{Conclusions}

In the present work, we have obtained a set of materials of UiO-66 type MOFs, with two linkers NDC and BDC - in various ratios; we have also investigated the effect of BA additives (in amount 10 and $60 \mathrm{eq}$ ) on the properties of the resulting materials. Moreover, as a further step, we used the post-synthetic technique of ligand exchange, to replace BA molecules in defect pores of UiOMOFs, by PhPA. Comprehensive characterization of obtained samples allowed us to make the following conclusions.

Summarizing the first part of the present work, we have observed that modulator additive to the system with mixed linkers leads to non-linear changes in the properties of materials as a result of several overlapping trends. First, injection of 10 eq of BA during the synthesis significantly enhances the porosity of obtained materials and leads to the formation of well-shaped separated nanoparticles instead of agglomerates (without BA) or large micro-meter crystals (with 60 eq of BA). Secondly, a high concentration of BA changes the NDC/BDC ratio in the resulting MOF relative concentration of NDC is higher than the stoichiometric value.

In the second part of the work, we used a post-synthetic exchange. Trends observed in the first part of work had a significant effect on this process. First, we have observed that samples obtained without BA did not contain PhPA molecules after exchange. So, we could claim that PhPA molecules exchange BA molecules in the defect pores. Secondly, we have observed that more massive NDC linkers hinder access to the cage and therefore obstruct exchange with BA molecules. We also have observed that an increase in SSA leads to a more successful exchange. Finally, using FTIR analysis and DFT calculations, we proved that PhPA molecules bond to the $\mathrm{Zr}^{4+}$ ions by $\mathrm{PO}_{4}$-groups instead of $\mathrm{COOH}$-groups. This means that samples after post-synthetic exchange contain defect pores decorated with free carboxylic groups.

\section{Acknowledgments}


The authors acknowledge RFBR for financial support according to project 18-29-04053.

\section{REFERENCES}

[1] H. Furukawa, O.M. Yaghi, Storage of Hydrogen, Methane, and Carbon Dioxide in Highly Porous Covalent Organic Frameworks for Clean Energy Applications, J. Am. Chem. Soc., 131 (2009) 8875-8883.

[2] W. Kleist, F. Jutz, M. Maciejewski, A. Baiker, Mixed-Linker Metal-Organic Frameworks as Catalysts for the Synthesis of Propylene Carbonate from Propylene Oxide and CO2, Eur. J. Inorg. Chem., (2009) 3552-3561.

[3] L.J. Murray, M. Dinca, J.R. Long, Hydrogen storage in metal-organic frameworks, Chemical Society Reviews, 38 (2009) 1294-1314.

[4] A. Corma, H. Garcia, F. Xamena, Engineering Metal Organic Frameworks for Heterogeneous Catalysis, Chem. Rev., 110 (2010) 4606-4655.

[5] V.V. Butova, M.A. Soldatov, A.A. Guda, K.A. Lomachenko, C. Lamberti, Metal-Organic Frameworks: Structure, Properties, Synthesis, and Characterization, Russ. Chem. Rev., 85 (2016) 280-307.

[6] V.V. Butova, M.V. Kirichkov, A.P. Budnyk, A.A. Guda, M.A. Soldatov, C. Lamberti, A.V. Soldatov, A room-temperature growth of gold nanoparticles on MOF-199 and its transformation into the $[\mathrm{Cu} 2(\mathrm{OH})(\mathrm{BTC})(\mathrm{H} 2 \mathrm{O})] n$ phase, Polyhedron, 154 (2018) 357-363.

[7] V.V. Butova, V.A. Polyakov, A.P. Budnyk, A.M. Aboraia, E.A. Bulanova, A.A. Guda, E.A. Reshetnikova, Y.S. Podkovyrina, C. Lamberti, A.V. Soldatov, Zn/Co ZIF family: MW synthesis, characterization and stability upon halogen sorption, Polyhedron, 154 (2018) 457-464.

[8] M. Eddaoudi, D.B. Moler, H.L. Li, B.L. Chen, T.M. Reineke, M. O'Keeffe, O.M. Yaghi, Modular chemistry: Secondary building units as a basis for the design of highly porous and robust metal-organic carboxylate frameworks, Accounts Chem. Res., 34 (2001) 319-330.

[9] O.M. Yaghi, M. O'Keeffe, N.W. Ockwig, H.K. Chae, M. Eddaoudi, J. Kim, Reticular synthesis and the design of new materials, Nature, 423 (2003) 705-714.

[10] D.J. Tranchemontagne, J.L. Mendoza-Cortes, M. O'Keeffe, O.M. Yaghi, Secondary building units, nets and bonding in the chemistry of metal-organic frameworks, Chem. Soc. Rev., 38 (2009) 1257-1283.

[11] J.H. Cavka, S. Jakobsen, U. Olsbye, N. Guillou, C. Lamberti, S. Bordiga, K.P. Lillerud, A new zirconium inorganic building brick forming metal organic frameworks with exceptional stability, J. Am. Chem. Soc., 130 (2008) 13850-13851.

[12] L. Valenzano, B. Civalleri, S. Chavan, S. Bordiga, M.H. Nilsen, S. Jakobsen, K.P. Lillerud, C. Lamberti, Disclosing the Complex Structure of UiO-66 Metal Organic Framework: A Synergic Combination of Experiment and Theory, Chem. Mat., 23 (2011) 1700-1718.

[13] Y.T. Huang, W.P. Qin, Z. Li, Y.W. Li, Enhanced stability and CO2 affinity of a UiO-66 type metal-organic framework decorated with dimethyl groups, Dalton Trans., 41 (2012) 9283-9285.

[14] Z. Chen, S.L. Hanna, L.R. Redfern, D. Alezi, T. Islamoglu, O.K. Farha, Reticular chemistry in the rational synthesis of functional zirconium cluster-based MOFs, Coord. Chem. Rev., 386 (2019) 32-49.

[15] M. Kandiah, M.H. Nilsen, S. Usseglio, S. Jakobsen, U. Olsbye, M. Tilset, C. Larabi, E.A. Quadrelli, F. Bonino, K.P. Lillerud, Synthesis and Stability of Tagged UiO-66 Zr-MOFs, Chem. Mat., 22 (2010) 6632-6640.

[16] S. Dissegna, P. Vervoorts, C.L. Hobday, T. Duren, D. Daisenberger, A.J. Smith, R.A. Fischer, G. Kieslich, Tuning the Mechanical Response of Metal-Organic Frameworks by Defect Engineering, J. Am. Chem. Soc., 140 (2018) 11581-11584.

[17] M. Kandiah, S. Usseglio, S. Svelle, U. Olsbye, K.P. Lillerud, M. Tilset, Post-synthetic modification of the metal-organic framework compound UiO-66, J. Mater. Chem., 20 (2010) 9848-9851. 
[18] M.J. Katz, Z.J. Brown, Y.J. Colon, P.W. Siu, K.A. Scheidt, R.Q. Snurr, J.T. Hupp, O.K. Farha, A facile synthesis of UiO-66, UiO-67 and their derivatives, Chem. Commun., 49 (2013) 94499451.

[19] V.V. Butova, A.P. Budnyk, K.M. Charykov, K.S. Vetlitsyna-Novikova, A.L. Bugaev, A.A. Guda, A. Damin, S.M. Chavan, S. Øien-ØDegaard, K.P. Lillerud, A.V. Soldatov, C. Lamberti, Partial and Complete Substitution of the 1,4-Benzenedicarboxylate Linker in UiO-66 with 1,4Naphthalenedicarboxylate: Synthesis, Characterization, and H2-Adsorption Properties, Inorg. Chem., 58 (2019) 1607-1620.

[20] V.V. Butova, A.P. Budnyk, A.A. Guda, K.A. Lomachenko, A.L. Bugaev, A.V. Soldatov, S.M. Chavan, S. Øien-ØDegaard, U. Olsbye, K.P. Lillerud, C. Atzori, S. Bordiga, C. Lamberti, Modulator effect in UiO-66-NDC (1, 4-naphthalenedicarboxylic acid) synthesis and comparison with UiO-67-NDC isoreticular metal-organic frameworks, Crystal Growth and Design, 17 (2017). [21] W.B. Liang, C.J. Coghlan, F. Ragon, M. Rubio-Martinez, D.M. D'Alessandro, R. Babarao, Defect engineering of UiO-66 for $\mathrm{CO} 2$ and $\mathrm{H} 2 \mathrm{O}$ uptake - a combined experimental and simulation study, Dalton Trans., 45 (2016) 4496-4500.

[22] G.C. Shearer, S. Chavan, S. Bordiga, S. Svelle, U. Olsbye, K.P. Lillerud, Defect Engineering: Tuning the Porosity and Composition of the Metal-Organic Framework UiO-66, Chem. Mat., 28 (2016) 3749-3761.

[23] C. Atzori, G.C. Shearer, L. Maschio, B. Civalleri, F. Bonino, C. Lamberti, S. Svelle, K.P. Lillerud, S. Bordiga, Effect of Benzoic Acid as a Modulator in the Structure of UiO-66: An Experimental and Computational Study, J. Phys. Chem. C, 121 (2017) 9312-9324.

[24] M. Taddei, When defects turn into virtues: The curious case of zirconium-based metal-organic frameworks, Coord. Chem. Rev., 343 (2017) 1-24.

[25] O.V. Gutov, M.G. Hevia, E.C. Escudero-Adan, A. Shafir, Metal-Organic Framework (MOF) Defects under Control: Insights into the Missing Linker Sites and Their Implication in the Reactivity of Zirconium-Based Frameworks, Inorg. Chem., 54 (2015) 8396-8400.

[26] O.V. Gutov, S. Molina, E.C. Escudero-Adan, A. Shafir, Modulation by Amino Acids: Toward Superior Control in the Synthesis of Zirconium Metal-Organic Frameworks, Chem.-Eur. J., 22 (2016) 13582-13587.

[27] G.R. Cai, H.L. Jiang, A Modulator-Induced Defect-Formation Strategy to Hierarchically Porous Metal-Organic Frameworks with High Stability, Angew. Chem.-Int. Edit., 56 (2017) 563567.

[28] M. Taddei, R.J. Wakeham, A. Koutsianos, E. Andreoli, A.R. Barron, Post-Synthetic Ligand Exchange in Zirconium-Based Metal-Organic Frameworks: Beware of The Defects!, Angew. Chem.-Int. Edit., 57 (2018) 11706-11710.

[29] P.P. Cui, P. Wang, Y. Zhao, W.Y. Sun, Fabrication of Desired Metal-Organic Frameworks via Postsynthetic Exchange and Sequential Linker Installation, Crystal Growth \& Design, 19 (2019) 1454-1470.

[30] A. Koutsianos, E. Kazimierska, A.R. Barron, M. Taddei, E. Andreoli, A new approach to enhancing the $\mathrm{CO} 2$ capture performance of defective UiO-66 via post-synthetic defect exchange, Dalton Trans., 48 (2019) 3349-3359.

[31] Z. Yin, S. Wan, J. Yang, M. Kurmoo, M.H. Zeng, Recent advances in post-synthetic modification of metal-organic frameworks: New types and tandem reactions, Coord. Chem. Rev., 378 (2019) 500-512.

[32] J.M. Taylor, K.W. Dawson, G.K.H. Shimizu, A Water-Stable Metal-Organic Framework with Highly Acidic Pores for Proton-Conducting Applications, J. Am. Chem. Soc., 135 (2013) 11931196.

[33] G. Guerrero, J.G. Alauzun, M. Granier, D. Laurencin, P.H. Mutin, Phosphonate coupling molecules for the control of surface/interface properties and the synthesis of nanomaterials, Dalton Trans., 42 (2013) 12569-12585.

[34] C. Queffelec, M. Petit, P. Janvier, D.A. Knight, B. Bujoli, Surface Modification Using Phosphonic Acids and Esters, Chem. Rev., 112 (2012) 3777-3807. 
[35] V. Petricek, M. Dusek, L. Palatinus, Crystallographic Computing System JANA2006: General features, Z. Kristall., 229 (2014) 345-352.

[36] H. Wu, Y.S. Chua, V. Krungleviciute, M. Tyagi, P. Chen, T. Yildirim, W. Zhou, Unusual and Highly Tunable Missing-Linker Defects in Zirconium Metal-Organic Framework UiO-66 and Their Important Effects on Gas Adsorption, J. Am. Chem. Soc., 135 (2013) 10525-10532.

[37] V.V. Butova, A.P. Budnyk, K.M. Charykov, K.S. Vetlitsyna-Novikova, C. Lamberti, A.V. Soldatov, Water as a structure-driving agent between the UiO-66 and MIL-140A metal-organic frameworks, Chem. Commun., 55 (2019) 901-904.

[38] A.I. Orlova, S.G. Samoilov, G.N. Kazantsev, V.Y. Volgutov, D.M. Bykov, A.V. Golubev, E.Y. Borovikova, Investigation of Zirconium Phosphate Zr-3(PO4)(4) during Heating, Crystallogr. Rep., 54 (2009) 431-438.

[39] V.A. Shkuropatenko, Sol-gel synthesis of NZP phosphates, Funct. Mater., 23 (2016) 92-97.

[40] G.D. Liu, Y.H. Lin, Electrochemical sensor for organophosphate pesticides and nerve agents using zirconia nanoparticles as selective sorbents, Anal. Chem., 77 (2005) 5894-5901.

[41] S.Z. Wang, W. Morris, Y.Y. Liu, C.M. McGuirk, Y. Zhou, J.T. Hupp, O.K. Farha, C.A. Mirkin, Surface-Specific Functionalization of Nanoscale Metal-Organic Frameworks, Angew. Chem.-Int. Edit., 54 (2015) 14738-14742.

[42] S.X. Lin, W.L. Pan, R.J. Niu, Y. Liu, J.X. Chen, W.H. Zhang, J.P. Lang, D.J. Young, Effective loading of cisplatin into a nanoscale UiO-66 metal-organic framework with preformed defects, Dalton Trans., 48 (2019) 5308-5314.

[43] M.B. Hay, S.C.B. Myneni, Structural environments of carboxyl groups in natural organic molecules from terrestrial systems. Part 1: Infrared spectroscopy, Geochim. Cosmochim. Acta, 71 (2007) 3518-3532.

[44] S.E. Cabaniss, I.F. McVey, Aqueous infrared carboxylate absorbances: Aliphatic monocarboxylates, Spectroc. Acta Pt. A-Molec. Biomolec. Spectr., 51 (1995) 2385-2395.

[43] M.J. Frisch, G.W. Trucks, H.B. Schlegel, G.E. Scuseria, M.A. Robb, J.R. Cheeseman, G. Scalmani, V. Barone, G.A. Petersson, H. Nakatsuji, X. Li, M. Caricato, A.V. Marenich, J. Bloino, B.G. Janesko, R. Gomperts, B. Mennucci, H.P. Hratchian, J.V. Ortiz, A.F. Izmaylov, J.L. Sonnenberg, D. Williams-Young, F. Ding, F. Lipparini, F. Egidi, J. Goings, B. Peng, A. Petrone, T. Henderson, D. Ranasinghe, V.G. Zakrzewski, J. Gao, N. Rega, G. Zheng, W. Liang, M. Hada, M. Ehara, K. Toyota, R. Fukuda, J. Hasegawa, M. Ishida, T. Nakajima, Y. Honda, O. Kitao, H. Nakai, T. Vreven, K. Throssell, J.A. Montgomery, Jr., J. E. Peralta, F. Ogliaro, M.J. Bearpark, J.J. Heyd, E.N. Brothers, K.N. Kudin, V.N. Staroverov, T.A. Keith, R. Kobayashi, J. Normand, K. Raghavachari, A.P. Rendell, J.C. Burant, S.S. Iyengar, J. Tomasi, M. Cossi, J.M. Millam, M. Klene, C. Adamo, R. Cammi, J.W. Ochterski, R.L. Martin, K. Morokuma, O. Farkas, J.B. Foresman, D.J. Fox, Gaussian 16, Revision A.03, Gaussian, Inc., Wallingford CT, 2016.

[44] A.D. Becke, Density-functional thermochemistry. III. The role of exact exchange, J. Chem. Phys., 98 (1993) 5648-5652.

[45] S. Grimme, S. Ehrlich, L. Goerigk, Effect of the damping function in dispersion corrected density functional theory, J. Comp. Chem., 32 (2011) 1456-1465. 